\title{
Global Workspace Theory Inspired Architecture for Autonomous Structural Health Monitoring
}

\author{
Mark M. Derriso \\ Air Force Research Laboratory \\ Air Vehicles Directorate \\ Wright-Patterson A.F.B., OH 45433
}

\author{
Charles D. McCurry \\ Booz Allen Hamilton \\ Dayton, OH 45420
}

\author{
Martin P. DeSimio \\ University of Dayton Research Institute \\ Dayton, OH 45469
}

\begin{abstract}
Structural health monitoring (SHM) systems provide automated assessments of structural health by processing data from sensors fastened to a structure. Most SHM research is focused on developing quick state assessments (i.e., reflexive techniques) using a form of pattern recognition. However, little attention has been given to developing a more 'thoughtful,' logicbased method for state assessments to enable operational-level decisions. Reflexive-based assessments are unreliable because they often cannot discriminate between changes due to structural damage and other variants (e.g., temperature). A new architecture is being proposed that combines reflexive and deliberative elements for enhanced state assessments and operational decisions. The architecture is demonstrated in the laboratory using a representative airframe component.
\end{abstract}

Keywords- structural health monitoring, intelligent agent, AFGROW, artificial nueral networks

\section{INTRODUCTION}

The Department of Defense (DoD) supports investigations across a variety of technologies aimed at reducing operational cost, increasing availability, and maintaining safety of current and future weapon systems. In fact, operations and support (O\&S) of DoD weapon systems accounts for 65 to $80 \%$ of the total lifecycle cost [1]. One of the principal contributors to $\mathrm{O} \& \mathrm{~S}$ cost is the vehicle maintenance process. The current procedure for maintaining U.S. Air Force air structures, the Aircraft Structural Integrity Program (ASIP), requires vehicles to be removed from service for routine maintenance, including inspection, at predetermined times regardless of their actual condition. This schedule-based maintenance approach works well for ensuring system integrity; however, it is very costly, labor-intensive, and reduces system availability. The costs are also continuously rising due to the frequent inspections required to maintain aircraft safety in aging fleets; aircraft in the U.S. Air Force have an average age of roughly 24 years.

SHM can be defined as automated methods for determining adverse changes in the integrity of mechanical systems. The ultimate goal of SHM is to provide an automated and real-time assessment of a structure's ability to serve its intended purpose. The need for and benefits of SHM systems for civil, military, and aerospace applications have been documented by many researchers [2]. Ideally, SHM would provide a diagnosis of the current state of a structure and a prognosis about the capability of the structure to perform its function in the future. The diagnosis should include the detection, localization, and assessment of any damage. The prognosis might be that the structure is as good as new; safe to operate for only a certain number of flight hours or that immediate repair is required. Many SHM experiments have been performed over the past decade, but few have transitioned to aerospace applications [3].

SHM systems are typically comprised of in-situ or embedded sensors and processing algorithms. The algorithms are used to interpret sensor responses to discriminate between different damage types in order to provide an accurate damage assessment and corresponding prognosis. Various processing steps may be performed by the SHM system to transform the data into different forms which enhance the damage assessment ability. Most SHM systems process sensory data using pattern recognition to classify structural states [4]. Development of SHM systems based on pattern recognition requires training data from all anticipated damage states and operational environments to be effective. The training data is used to design a classifier and the resulting performance is evaluated by scoring the classification results from data not utilized during the design or training phases.

SHM systems provide real-time automated assessments of structural health by processing data from sensors fastened to a structure. The integrity of a structure can be assessed at different levels of detail. The two fundamental levels, detection of damage and its location, are useful in their own right. Using just results from the damage detection and localization levels, inspection time and costs could be reduced. However, information provided by the two higher levels of SHM, relating to quantifying the degree of damage and ultimately an assessment of the consequences of damage in 


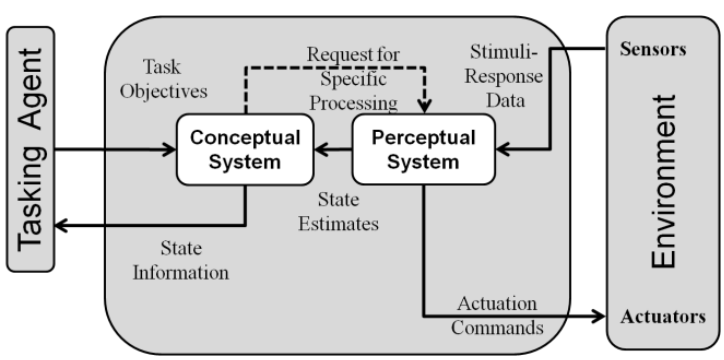

Fig. 1. General intelligent agent.

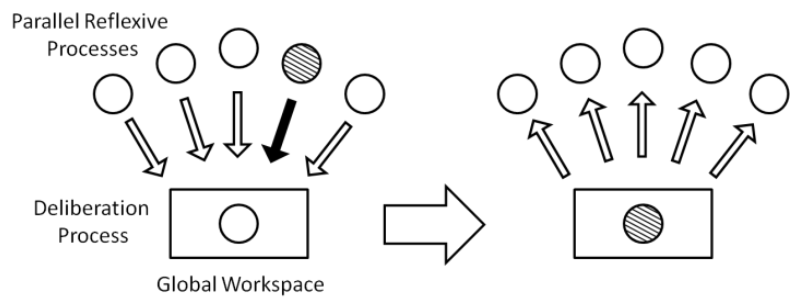

Fig. 2. Global Workspace Theory Architecture.

terms that are meaningful to maintainers, operators, and commanders - could lead to further improvements in operation.

\section{BACKGROUND}

\section{A. Intelligent Agents}

There are several definitions in the literature defining intelligent agents. This paper uses the definition proposed by Michael Wooldridge. Wooldridge defines an agent as a computer system that is situated is some environment, and that is capable of autonomous action in the environment in order to meet it delegated objectives [5]. The intelligence that an agent needs is directly related to its assigned task.

For example, a simple reflex agent can work well in a well-defined setting. Consider a thermostat as a simple agent. The sensor could be a thermocouple, and the actuator a heat pump. Condition-action rules can be in the form of "if-then" statements. In this case the rules may be: if (temperature $>$ T_hi), then set heat pump to cool; otherwise, if (temperature < T_low), then set heat pump to heat. The intelligence embedded in these simple rules can work well in controlled settings. However, in a more complex problem, such as in an SHM task, the intelligence of an agent may need to increase to provide the desired levels of performance.

\section{B. Intelligent Agents Architectures}

Architectures are the blueprints for intelligent agents. They are useful in acquiring and processing sensory data. This information is then used to perform the appropriate action to achieve the assigned task. Fig. 1 shows an overview of a general intelligent agent architecture. The tasking agent supplies goals and objectives that can be mapped to conditionaction rules in the conceptual system. The conceptual system receives estimated states from the perceptual system, and uses context information and goal-based reasoning to provide state information to the tasking agent. The conceptual system can also command the perceptual system to collect additional data or perform other processing steps as specified in the condition-

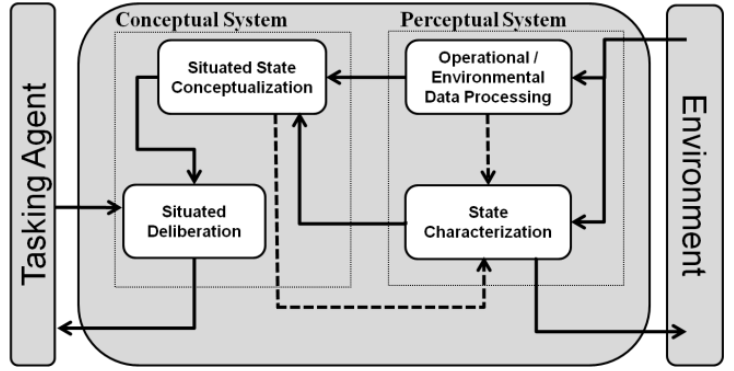

Fig. 3. Detailed view of intelligent agent.

action rules. The perceptual system interacts with the environment and provides state estimates to the conceptual system.

The Global Workspace Theory (GWT) is an architecture that includes a particular blend of serial and parallel computations for processing information. It was developed by Bernard Baars to account qualitatively for a large set of matched pairs of reflexive and deliberative processes [6]. The principle theory of this architecture is the multiple parallel specialized processes that compete and cooperate for access to the global workspace where the information is fused, processed, and deliberated before a conclusion is reached. In addition, the global workspace could request specific queries of the parallel processes if necessary for disambiguation. Fig. 2 shows a graphical representation of the GWT architecture. Since Baars only gives a metaphorical description of the GWT, more engineering design is required before this architecture is realized for specific applications.

Fig. 3 shows a more detailed representation of an intelligent agent architecture inspired by the GWT. The conceptual system consists of two main components: situated state conceptualization and situated deliberation. The perceptual system also consists of two main components: operational/environmental data processing and state characterization. Each of the main blocks operates as follows.

The operational and environmental processing block accepts measurement data that gives context regarding how the system is being operated and the environment that the system is operated in. For example, temperature is environmental data, and load levels and load cycles are operational data.

State characterization is the process used to estimate health status from sensor data. The dashed line entering the top of the state characterization box indicates that operational/environmental data could be used to supply context. For example, temperature compensation could be applied to the SHM sensor data based on thermocouple readings. The output of the state characterization stage is one or more estimates of the state of the monitored system. State estimates at this stage give information related to presence of damage, and the degree of damage, such as crack length estimates for a monitored location on a vehicle.

Situated state conceptualization involves using context information and/or physics-based models to refine state estimates. Context is provided by the operational and environmental block. Load data can be used in a physics-based 


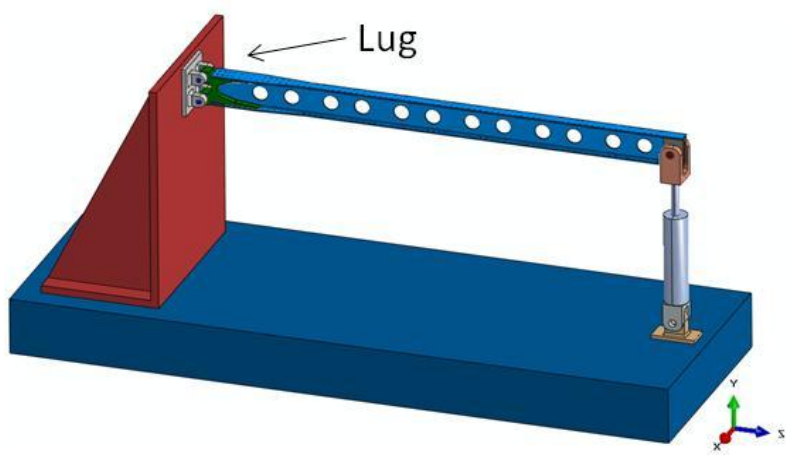

Fig. 4. Representative wing attachment lug in test bed.

model to predict the existence of a crack given knowledge of the material properties and geometry of the part. Additionally at the situated state conceptualization stage, requests for specific processing can be made, as indicated by the dashed line to the state characterization block.

The specific processing may be as simple as requesting repeated measurements from the same sensors, or even computing state estimates from secondary methods that are possibly more computationally demanding. The state estimate provided by the situated state conceptualization stage makes use of predetermined condition-action rules designed to satisfy the goals of the tasking agent.

Finally, the situated deliberation stage provides a goaloriented action to the tasking agent that is based on context information as well as state estimates. The goal-oriented actions will differ based on the needs of the particular tasking agent. For example, a maintainer may design rules to be notified when a visual damage inspection is needed. Or, a mission commander my design rules that report risk of component or mission failure for the given current structural state and anticipated flight profile.

The intelligent agent architecture described above has been applied to a laboratory demonstration test article, and is described in the following section.

\section{EXPERIMENT}

The intelligent agent architecture has been applied to a representative aircraft component, and generates context-based decisions from state estimates made crack detection and length estimation models. The algorithm estimates are combined with a physics-based crack growth model. The tasking agent is supplied with a risk of component failure using a given flight profile and estimated remaining life of the component.

The representative component is a wing attachment lug. It is part of an assembly mounted in a test bed and loaded with an actuator as shown in Fig. 4. The lug is milled from 6061-T6 aluminum alloy. One end of the lug is attached to the test bed using a clevis and mounting pins. The other end of the lug is attached to an aluminum beam representing a wing spar. The far end of the beam is driven by a hydraulic actuator. The loading will eventually lead to crack initiation and growth in the shoulders of the lug. The shoulder regions of the lug were instrumented using bonded Kapton-encapsulated piezoelectric

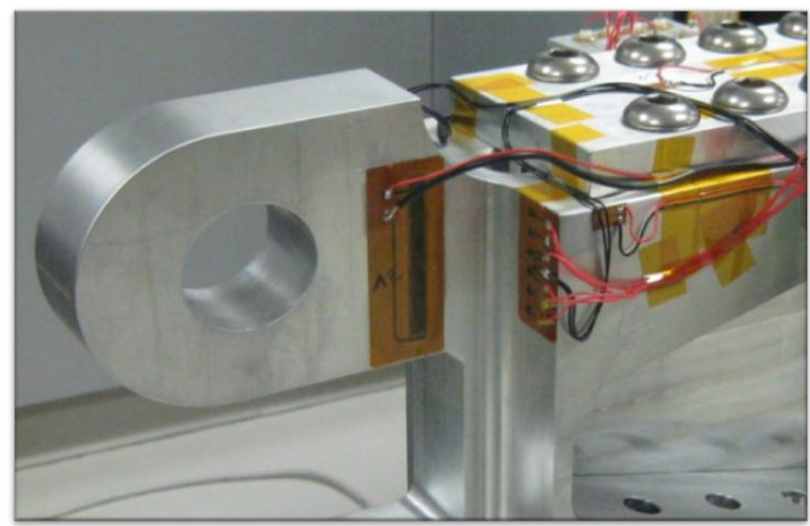

Fig. 5. Piezoelectric transducers mounted on lug.

transducers (PZTs) as shown in Fig. 5. After installation in the test bed, the lug was loaded for a predetermined number of cycles, and then paused for SHM and visual crack length measurements. During the SHM measurements, ultrasonic elastic waves are transmitted through the shoulder region, from a rectangular actuation PZT to six sensing PZT disks. The actuation signals are $5 \frac{1}{2}$ cycle windowed tone bursts with center frequencies ranging from $400 \mathrm{kHz}$ to $1 \mathrm{MHz}$ in $100 \mathrm{kHz}$ steps. Responses are recorded with a $10 \mathrm{MHz}$ sample rate and 12-bit amplitude resolution.

The fatigue life of the lug has been estimated using AFGROW, a crack growth software model developed by the U.S. Air Force. Assuming an initial flaw size of 0.02", under constant amplitude sinusoidal loading between 0 and 1000 pounds, the lug is predicted to fail after 14500 cycles.

Measurements were conducted during pauses in the fatigue cycling. The interval between inspections was based on the visual crack length. Measurements were made every 1000 cycles until a crack was visually detected. After visual detection, measurements were made every 500 cycles until the longest observed crack reached 0.3". Once the longest crack reached 0.3 ", measurements were made every 250 cycles. The experiment was terminated when the longest crack reached 0.7'. This schedule provided 123 measurements over 70000 fatigue cycles. One primary factor of the discrepancy between the estimated and observed fatigue life is the uncertainty involved with crack initiation. The AFGROW model assumes a 0.02 " crack existed in the part at the beginning of the test; in practice, the initial flaw was likely smaller than 0.02 ".

\section{A. SHM State Estimation}

The fundamental feature for crack length estimation is based on a damage index derived from the correlation coefficient between reference and test measurements. The reference measurements are from the cycle 1000 trial. A damage index is computed at each sensor for each tone burst frequency. The damage index is defined to be $\left(1-\rho_{x y}\right)$, where $\rho_{x y}$ is the correlation coefficient between a segment of the reference and corresponding segment of the test signal. Segments are specified to include essentially the interval around the first arriving packet; signals are shown in Fig. 6. 


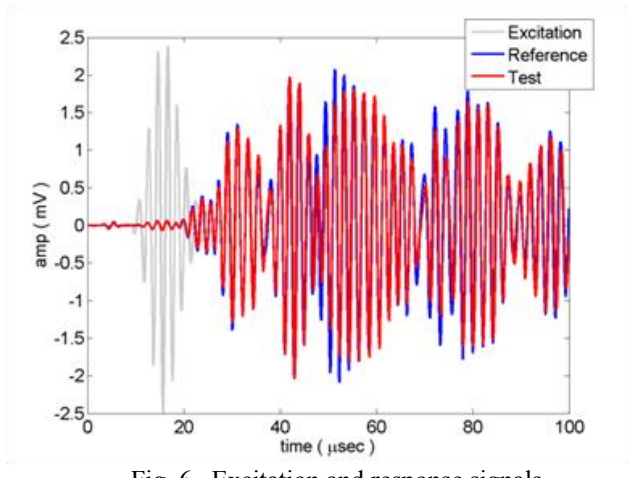

Fig. 6. Excitation and response signals.

A linear regression model for mapping damage indices to visual crack length measurements was designed using data from the completed test. The data were randomly divided into training and test partitions across the experimental signal collections. For the six sensors and seven frequencies, 42 damage index values are computed at each SHM measurement. Feature selection is based on a stepwise regression procedure [7]. The procedure involves iteratively fitting a series of multilinear regression models to crack length measurements using different subsets of elements from the feature vector. The subset of features grows or shrinks based on the significance of a feature's contribution to the regression model. A feature is added to the subset only when its presence in the model improves the fit. Conversely, a feature is removed from the subset when its absence does not degrade the fit. The stepwise procedure terminates when the addition of any remaining feature does not improve the fit and the removal of any previously selected feature degrades the fit.

Another estimation model was also developed using an artificial neural network (ANN). The ANN was trained using the damage index values and visual crack length measurements. As with the regression modeling, the data was randomly divided into training and test partitions across the experimental signal collections. Unlike the regression modeling, the ANN does not use features extraction; thus all 42 damage index values were used in its development.

\section{B. Physics-based crack length modeling}

As mentioned above, AFGROW is software that was developed by the U.S. Air Force and performs fracture mechanics and fatigue crack growth analyses. The software uses a current crack length, along with material properties, part geometry, and loading information to compute the number of cycles remaining before the crack reaches the critical crack length, resulting in failure. Fig. 7 shows estimated crack lengths versus load cycles for various peak loads.

\section{Situated State Conceptualization}

Situated State Conceptualization uses current crack length estimates (estimated states) and an AFGROW predicted crack length (predicted state), calculated from the previous selected state, to determine the current selected crack state through use

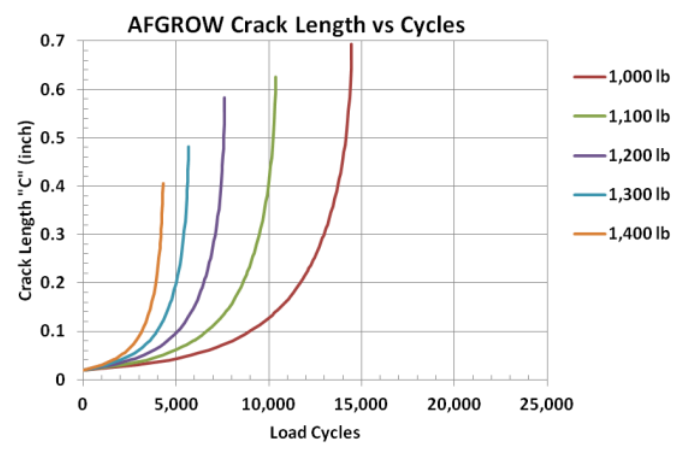

Fig. 7. Model predicted crack lengths.

of a percentage agreement criterion condition-action rule base algorithm. A discussion of how AFGROW is used to predict crack state and the percentage agreement criterion conditionaction rule base algorithm follows.

Fig. 8 shows the AFGROW curve for $1000 \mathrm{lb}$ loading. In Fig. 8, SS(n-1) is the previous selected state, and from $\mathrm{SS}(\mathrm{n}-1)$ on the curve, $\mathrm{CYC}(\mathrm{n}-1)$ is determined. This value is taken as a starting point for subsequent crack growth. Since the elapsed cycle count between measurements is known, the predicted crack state is found by adding $\triangle \mathrm{CYC}$ to $\mathrm{CYC}(\mathrm{n}-1)$. The predicted crack state, $\mathrm{PS}(\mathrm{n})$, is found by reading the growth curve of Fig. 8 at $\mathrm{CYC}(\mathrm{n})=\mathrm{CYC}(\mathrm{n}-1)+\Delta \mathrm{CYC}$.

The percentage agreement criterion condition-action rule base is as follows: if the first two estimated states are greater than the previous selected state and are within a certain percentage of each other, then their average is used as the current selected state. If they are not in agreement with each other, then each are checked individually for agreement with the predicted state. This can result in the selected state being an average of either one of the crack estimated states and the predicted state, or an average of all three.

If an agreement is not reached by this point, the situated state conceptualization will request two additional estimated states and the agreement criterion process repeats; if an agreement is not reach at the end of this phase, the selected state is defaulted to the AFGROW predicted state. The percentage agreement criterion condition-action rule base algorithm is shown in detail in Fig. 9.

The algorithm is based on the assumption that the estimation techniques are able to detect a crack initiation of equal to or greater than 0.02 ". If the estimations do not detect crack initiation, then the AFGROW prediction portion of the Situated State Conceptualization is not activated, resulting in a continuous sequence of selected state crack lengths of 0 ". Fig. 10 plots the four estimated states, the predicted state and the visual crack measurements from the laboratory experiment and a MATLAB implementation of the Situated State Conceptualization steps. The crack initiates at 42000 cycles. Prior to 42000 cycles, there is no crack and the AFGROW predicted state is 0 ", and is also the selected state. At 42000 cycles, the first two estimates are neither in agreement with each other nor the predicted state. The algorithm requests additional information: the second two estimates. These two estimates are in agreement and determine crack initialization. 


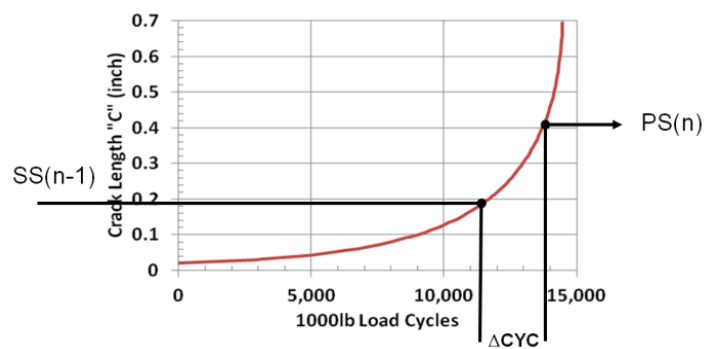

Fig. 8. Predicted current crack length given prior state and cycle count.

TABLE I

Estimates Selected for Averaging

\begin{tabular}{|c|c|c|c|c|c|} 
Cycle $(n)$ & $N N_{1}(n)$ & $R E G_{1}(n)$ & $N N_{2}(n)$ & $R E G_{2}(n)$ & $P(n)$ \\
\hline 41000 & & & & & $\mathrm{X}$ \\
\hline 41500 & & & & & $\mathrm{X}$ \\
\hline 42000 & & & $\mathrm{X}$ & $\mathrm{X}$ & \\
\hline 42500 & $\mathrm{X}$ & $\mathrm{X}$ & & & \\
\hline 43000 & $\mathrm{X}$ & $\mathrm{X}$ & & & $\mathrm{X}$ \\
\hline
\end{tabular}

The average of the states in agreement is used as the selected state, and these are shown marked by X's in Table I. One can also see in Table 1 at the next cycle of 42500 , that the first two estimates are now in agreement, thus no request for additional estimates is needed and their average is used as the selected state. At cycle 43000 , the first two estimates and the predicted state are in agreement, and their average is used as the selected state. Fig. 11 shows the visual crack measurements and selected crack states resulting from applying the rule base described in Fig. 9.

\section{Situated Deliberation}

The objective of the situated deliberation stage is to determine the cycles remaining to failure of the component based on the current crack state. Refer again to the $1000 \mathrm{lb}$ load curve of Fig. 7, which shows the relationship between load cycles and crack length. In the case of situated deliberation, the crack state computed by AFGROW is used to estimate the number of remaining cycles. That is, this figure can be interpreted as showing the estimated remaining cycles before failure versus the current crack state. For example, consider entering the graph from the vertical axis at the crack length of 0.2". The AFGROW estimated cycle count corresponding to this crack length is approximately 11800 cycles. Additionally, the number of cycles to failure has already been computed by AFGROW, and is 14500 cycles. Therefore, the estimated remaining life of the part is found by subtraction to be 2700 cycles. Assignment of risk of mission failure cannot be determined until a mission profile is specified. However, assume that risk categories are arbitrarily assigned to low, medium, and high values based on the remaining life.

For the sake of demonstration, consider a future mission profile of 4000 cycles. For this case, assume a commander defines low risk when the estimated remaining cycles to failure is over 5000, medium when the estimated remaining cycles to failure is between 3000 and 5000, and high when the estimated remaining cycles to failure is under 3000. Fig. 11 plots the estimated remaining cycles for the situated state conceptualization selected state (blue), visual crack measurement (black) and the AFGROW selected state (red), after crack initialization was determined. Consider the situation where the component has operated for 40000 cycles.

The mission commander now asks "What is the risk involved in completing a future mission profile of 4000 cycles at the $1000 \mathrm{lb}$ load?" The intelligent agent architecture can be used to answer this question. At the 40000 cycle count, the crack size for the situated state conceptualization selected state, visual crack measurement and AFGROW selected state is 0". The estimated number of remaining cycles for the component by all three methods at the 40000 cycle count is approximately 14500 cycles. Thus the situated deliberation outcomes for the three methods are mapped to the low risk category.

Next, assume the part has operated to the 45000 cycle count. Based on just the AFGROW selected state at this cycle count, the estimated number of remaining cycles is approximately 5000 cycles. This situated deliberation outcome gets mapped to the medium risk category. For the situated state conceptualization selected state the estimated number of remaining cycles is approximately 7000 cycles, and approximately 6500 cycles for the visual crack measurement. Both of these situated deliberation outcomes get mapped to the low risk category. Finally, assume now that the part has undergone 50000 cycles. The estimated number of remaining cycles is zero for the AFGROW selected state. This situated deliberation outcome gets mapped to the high risk category. The visual crack measurement and situated state conceptualization selected state estimated number of remaining cycles is about 5000 cycles. These situated deliberation outcomes both get mapped to the medium risk category.

What is more interesting to note in Fig. 12 is that upon crack initiation around 42000 cycles, AFGROW, which does not use the actual crack length to update itself and is solely based on the initial crack size once detected, predicts that the component fails at the 50000 cycle count, or about 8000 cycles later. However, the visual crack measurement shows that the component actually does not fail until about the 67000 cycle count or 25000 cycles later. Note that the situated state conceptualization selected state tracks the visual measurement more closely than the AFGROW estimates. This architecture offers the potential of providing the mission commander a real-time assessment of the actual capability of the component.

\section{SUMMARY}

This paper presents an intelligent agent architecture, inspired by the Global Workspace Theory, that combines reflexive and deliberative elements to support enhanced state assessments and operational decisions. The intelligent agent architecture is realized using MATLAB/Simulink software, and is demonstrated using data recorded in a laboratory experiment with a representative airframe component, a wing 
Variable Description

Four estimation states: $E_{1}(n), E_{2}(n), E_{3}(n), E_{4}(n) \quad$ Percentage value: $\pm \% \mathrm{X}$

Predicted state: $P S(n)$

Selected state: $S S(n)$

Previous state $S S(n-1)$.

1.) If $E_{1}(n)$ and $E_{2}(n)$ are $\left.\geq S S(n-1)\right)$ and $E_{1}(n)$ and $E_{2}(n)$ are within $\pm \% \mathrm{X}$ of each other $\left\{\right.$ i.e. $E_{1}(n)-P * E_{1}(n) \leq E_{2}(n) \leq$ $E_{1}(n)+P * E_{1}(n)$ and $\left.E_{2}(n)-P * E_{2}(n) \leq E_{1}(n) \leq E_{2}(n)+P * E_{2}(n) \quad\right\}$ then $S S(n)=\frac{E_{1}(n)+E_{2}(n)}{2}$

2.) If $E_{1}(n)$ is $\left.\geq S S(n-1)\right)$ and $E_{1}(n)$ is within $\pm \% \mathrm{X}$ of $P S(n)$, then $S S(n)=\frac{E_{1}(n)+P S(n)}{2}$

3.) If $E_{2}(n)$ is $\left.\geq S S(n-1)\right)$ and $E_{2}(n)$ is within $\pm \% \mathrm{X}$ of $P S(n)$, then $S S(n)=\frac{E_{2}(n)+P S(n)}{2}$

4.) If $E_{1}(n)$ and $E_{2}(n)$ are $\left.\geq S S(n-1)\right)$ and $E_{1}(n)$ and $E_{2}(n)$ are within $\pm \% \mathrm{X}$ of $P S(n)$, then $S S(n)=\frac{E_{1}(n)+E_{2}(n)+P S(n)}{3}$

5.) If the first four steps do not yield the selected state, the architecture feedbacks to select two additional estimations or $E_{3}(n)$ and $E_{4}(n)$

6.) If $E_{3}(n)$ and $E_{4}(n)$ are $\left.\geq S S(n-1)\right)$ and $E_{3}(n)$ and $E_{4}(n)$ are within $\pm \% \mathrm{X}$ of each other then $S S(n)=\frac{E_{3}(n)+4(n)}{2}$

7.) If $E_{3}(n)$ is $\left.\geq S S(n-1)\right)$ and $E_{3}(n)$ is within $\pm \% \mathrm{X}$ of $P S(n)$, then $S S(n)=\frac{E_{3}(n)+P S(n)}{2}$

8.) If $E_{4}(n)$ is $\geq S S(n-1)$ ) and $E_{4}(n)$ is within $\pm \% \mathrm{X}$ of $P S(n)$, then $S S(n)=\frac{E_{4}(n)+P S(n)}{2}$

9.) If $E_{3}(n)$ and $E_{4}(n)$ are $\left.\geq S S(n-1)\right)$ and $E_{3}(n)$ and $E_{4}(n)$ are within $\pm \% \mathrm{X}$ of $P S(n)$, then $S S(n)=\frac{E_{3}(n)+E_{4}(n)+P S(n)}{3}$

10.) If the selected state is not determined by this point then $S S(n)$ is set equal to $P S(n)$.

Fig. 9. Percentage Criterion Condition-Action Rule Base for Situated State Conceptualization.

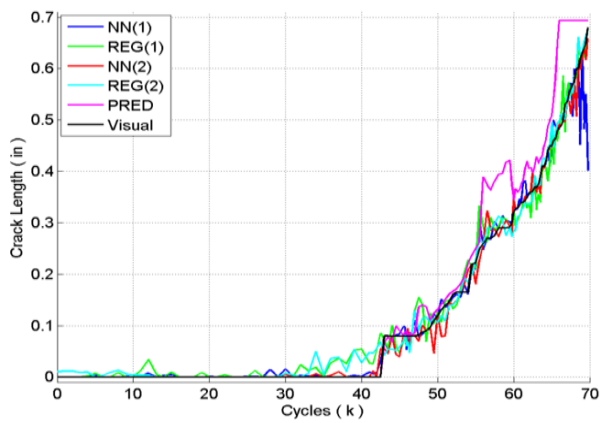

Fig. 10. Model predicted crack lengths.

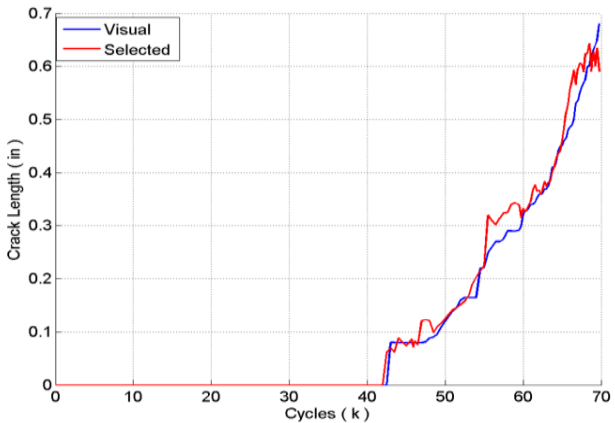

Fig. 11. Selected crack length versus cycle.

lug, instrumented with piezoelectric transducers. Reflexive algorithms, regression modeling and artificial neural networks, within the architecture are combined with a deliberative condition-action rule base algorithm element to provide a state assessment with respect to lug crack existence and length estimate from the piezoelectric sensory data. Using the crack state assessment and AFGROW software, the intelligent agent architecture determines the operational risk related to use of the lug for a future mission profile.

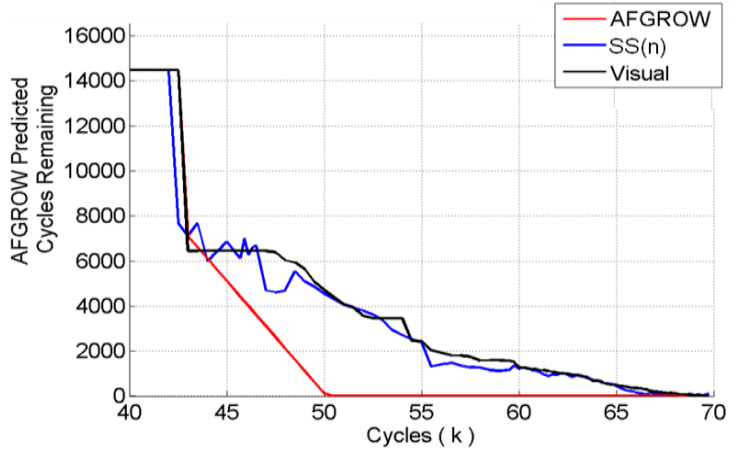

Fig. 12. Predicted cycles remaining versus current cycles.

\section{ACKNOWLEDGMENT}

A portion of this work was supported by a grant from AFOSR's Multi-Scale Structural Mechanics and Prognosis Program. The authors are grateful to Todd Bussey of Sierra Lobo for his expertise in instrumentation and data acquisition.

\section{REFERENCES}

[1] Department of Defense, Condition-Based Maintenance Plus DoD Guidebook, May 2008.

[2] H. Sohn et al, "A Review of Structural Health Monitoring Literature: 1996-2001," LA-13976, Los Alamos National Laboratory, 2004

[3] M. Derriso et al., "Why are there few fielded SHM systems for aerospace structures?", in Proceedings 2007 International Workshop on SHM, Stanford, CA, 2007

[4] K. Worden et al, "The fundamental axioms of structural health monitoring,” Proc. R. Soc. A, Vol. 463, No. 2083, pp. 1639-1664, 8 June 2007.

[5] M. Wooldridge, Reasoning about Rational Agents, The MIT Press, 2000

[6] M. Shanahan, B. Baars, "Applying global workspace theory to the frame problem," Cognition, pp. 157-176, 2005.

[7] N. Draper and H. Smith, Applied Regression Analysis, $2^{\text {nd }}$ Ed., J. Wiley \& Sons, 1981. 